\title{
Restoring the Function of a Diseased Kidney via Its Microvasculature
}

\author{
Leon G. Fine \\ Cedars-Sinai Medical Center and University of California at Los Angeles, Los Angeles, Calif., USA
}

\section{Key Words}

Microvasculature $\cdot$ Regeneration $\cdot$ Nephron

\begin{abstract}
Background: Based upon observations which indicate that chronic intrarenal hypoxia and microvascular obliteration play an important role in the pathogenesis of renal scarring and loss of function, the idea is presented that restoration of kidney structure and function by arresting microvascular drop-out and restoring the interstitial capillary network could be a feasible approach to regeneration of a diseased kidney. This paper addresses the reasoning behind this possibility. Summary: A 'unifying vasculogenic hypothesis' is discussed which proposes that, in hypoxic nephrons which retain poorly functioning vascular and epithelial elements, the disease process can be slowed or arrested, and nephrons regenerated, by adoptive transfer of endothelial progenitor cells to restore interstitial and glomerular vascular integrity. It is suggested that no other cell types are required to achieve this end. Improved differentiation, proliferation, and function of surviving nephrons could be achieved by restoring adequate oxygen delivery via this approach. Key Messages: It is hypothesized that, to regenerate the function of a chronically diseased kidney, it is not plausible to create new nephrons. Restoration of function of surviving nephrons could be achieved by regeneration of the renal microvasculature
\end{abstract}

alone. Based upon observations that have demonstrated the feasibility of adoptive endothelial progenitor cell transfer into the kidney, this hypothesis is worthy of being tested.

(c) 2014 S. Karger AG, Basel

\section{To Regenerate or Not to Regenerate}

There appear to be only two options for regenerating a chronically diseased kidney: one would be to create new nephrons and integrate these into the vascular supply and collecting system of the native kidney and the second would be to resurrect partially functioning nephrons in the diseased kidney and attempt to restore as much function as possible to these.

The first option is too daunting to contemplate. The human nephron has a vascular capillary tuft at one end connected to a high-pressure arterial system so that it can act as a filtration device; it has at least 30 phenotypically different cell types along the length of its tubule and it forms branched connections to other nephrons as it drains into the collecting system. Even if a multipotent 'renal cell' could be isolated and coaxed into multiple lineages of differentiation, achieving the architectural challenges required to construct a new nephron seems to be impossible outside of the normal developmental program of the organ.

\section{KARGER}

E-Mail karger@karger.com

www.karger.com/nee
(C) 2014 S. Karger AG, Basel

$1660-2129 / 14 / 1262-0082 \$ 39.50 / 0$
Leon G. Fine, MD

Cedars-Sinai Medical Center and University of California at Los Angeles 8700 Beverly Blvd. Davis 5093

Los Angeles, CA 90048 (USA)

E-Mail leon.fine@cshs.org 
The second option, i.e. regenerating surviving nephrons, seems to be the most feasible one. Taking this approach, it would be necessary to begin the process at a time when the functioning nephron population is depleted but not when the kidney is totally scarred and shrunken as occurs in all forms of end-stage renal disease.

What makes this possibility attractive? First is the fact that insights based upon exquisitely performed microdissections of diseased nephrons by Jean Oliver [1] in the 1930s indicate that there remain in the diseased kidney nephrons which are distorted in shape and size, retain their connections to the glomerular filters, and yet possess tubular cells which are flattened and presumably still functioning, albeit poorly. The 'skeleton' of the nephron remains, meaning that its longitudinal structure, provided by a tubular basement membrane and a glomerular capsule, is available to be used as a scaffold.

\section{The Central Role of the Microvascular Insufficiency and Progression of Kidney Disease}

An additional important histopathological finding in chronically diseased kidneys is the drop-out and disappearance of interstitial capillaries throughout the cortex and medulla observed in human renal biopsies [2]. There are a number of proposed causes for the progressive nature of most renal diseases, and it is probable that no single cause predominates. We have tried to address the mystery of why an initial insult to the kidney (in the absence of an inciting cause, such as an infectious agent) does not simply heal or cause a scar of limited dimensions. Rather the scarring becomes extensive until the whole organ is consumed.

\section{Chronic Hypoxia as a Mechanism for Progressive Tubulointerstitial Fibrosis}

We have proposed a 'chronic hypoxia hypothesis' to explain a series of events which can result in an inadequate delivery of oxygen to the interstitium of the kidney [2-4]. This hypothesis argues that a primary glomerular disease, which involves most of the capillaries within a glomerulus, will impair blood flow through the glomerulus and that blood flow into the efferent arteriole is accordingly decreased. This means that blood flow into the surrounding interstitial compartment is also reduced (since all interstitial capillaries derive from the efferent arteriole). This leads to patchy regions of hypoxia within the kidney. Based upon in vitro observations, we have shown that hypoxia per se is 'fibrogenic' and causes the accumulation of interstitial matrix material [3]. In vivo interventions which dilate the efferent arterioles (ACE inhibitors) improve renal oxygen tension [5]. The validity of this hypothesis has been supported by an increasing number of experimental observations [6].

Translated into an in vivo situation, this means that if the initial hypoxia is sufficiently severe it leads to the formation of a scar. When scars heal they obliterate the microvessels within them (pink scars turn into white scars), leading to more hypoxia surrounding the scar. This sets up a vicious cycle of expanding fibrosis and the resultant widening of obliteration of microvessels deletes the outflow tracts of glomeruli, resulting in a progressive fall of the filtration rate. Understanding this series of events is key to developing a strategy for regeneration as described below.

\section{Regeneration by Introducing Progenitor or Stem Cells Is Not Feasible unless the Original Disease of the Kidney Can Be Halted or Slowed}

Enthusiasts who have isolated a renal progenitor or stem cell and who believe that all that is needed is to find a way to introduce such cells into the kidney for regeneration to begin will fail to address an overarching reality: if there is a progressive downhill disease process taking place, it will destroy any new cells introduced into the affected environment. This includes resident progenitor cells, which could not be called upon to carry out the regenerative process required. This latter insight was provided by elegant studies in acutely injured kidneys, where it was found that resident renal progenitor cells become dysfunctional in the same way that differentiated cells do [7]. For this reason, the regenerative process cannot proceed spontaneously. Should extrinsic cell types be introduced into such a diseased environment, they would be subjected to the same fate as those of the native organ.

\section{Essential Requirements for Regeneration of a Chronically Diseased Kidney}

Based upon the above considerations it would seem necessary to find a means to initiate an intervention well in advance of end-stage disease, when an adequate number of surviving nephrons remain. It would be necessary to initiate a series of events which halt or slow down the 
existing disease process and, at the same time, to restore the microvessels, which would be a logical way of achieving an improved delivery of oxygen to areas undergoing fibrotic change.

In 2010, we outlined a unifying vasculogenic hypothesis for renal regeneration [8] and further elaborated on this in 2012 [9]. The essentials of this hypothesis are:

(1) Oxygen delivery to previously hypoxic nephrons can be achieved by restoring the interstitial microvasculature. Improved oxygen delivery will slow or halt the ongoing disease process.

(2) Adoptive transfer of endothelial progenitor cells could participate in the reconstruction of microvessels in the interstium and in the glomeruli. No cells other than progenitor endothelial cells would be required to achieve survival and regeneration of all elements within the kidney.

(3) Improved differentiation, proliferation, and function of surviving cells on existing scaffolds (glomerular and tubular) would result. Function would also be restored to dysfunctional resident stem cells which would participate in the regenerative process.

\section{Feasibility of Achieving Renal Regeneration via Its Microvessels}

One way of initiating the process of vasculogenesis (new vessel formation) would be to adoptively transfer endothelial precursors into the kidney $[7,10,11]$. In an experimental mouse model of Adriamycin-induced nephropathy, endothelial precursors injected systemically homed to the areas of injury and inflammation. This resulted in increased vascular density and improved overall function [7]. In a murine model of chronic kidney disease, such an intervention reduced glomerulosclerosis and interstitial fibrosis and improved the overall renal function [12]. In a model of renal artery stenosis with established microvascular disease, an improvement in capillary proliferation occurred [13].

In acute models, it may be that progenitor cells exert an anti-inflammatory effect or work through paracrine effects or via cell-cell contacts. However, the most intriguing possibility is that, through the formation of new vessel segments which integrate with host vessels to form chimeric vessels, the net effect would be to improve tissue oxygenation. Importantly, this would work to reverse the dysfunction of resident stem cells, too [14]. The net effect would be that more than one cell type would ultimately participate in healing and regeneration. Where progeni- tor cells have the ability to differentiate along different lineages, e.g. in the heart [15], it seems that it is the endothelial lineage, rather than cardiomyocyte lineage, that has the predominant effect in improving function.

The improved blood supply to the surviving nephron population would allow the flattened epithelial cells which line such nephrons to restore their original phenotypic features and regain function in an organized and integrated manner. The same would apply to glomerular epithelial cells, both parietal and visceral. The fact that renal oxygenation can be improved by vasoactive agents in an acute in vivo setting [5] suggests that a maneuver which restores the integrity of the interstitial capillaries in a sustained manner should improve oxygenation in the setting of chronic disease. It remains to be tested whether such an approach alone could restore poorly functioning nephrons and arrest their further deterioration.

\section{Learning from Bioengineering}

In a particularly impressive experimental feat, Song et al. [16] recently described their successful bioengineering of rat, pig, and human kidneys which had been decellularized to leave only the acelllular scaffolds of vascular, tubular, and collecting system structures in place. By seeding such scaffolds with endothelial and epithelial cells and perfusing these organs in a whole-organ bioreactor, they were able to achieve rudimentary function (urine formation) in vitro. When transplanted in the orthotopic position (in the rat) the kidney grafts, perfused by the recipients' circulations, produced urine via the ureteral conduit.

In the context of the present discussion, it is worth considering the vascular elements involved in this study [16]. Human umbilical vein endothelial cells were instilled via the renal artery. After 3-5 days these cells came to line the vasculature throughout the kidney, from large vessels to glomeruli to capillaries. Importantly, the engrafted cells only homed to their appropriate vascular compartments. Even in the glomeruli, where the capillaries are lined by both endothelial and epithelial cells, cell markers revealed these cell types to be in their appropriate locations.

Aside from the possible utility of this technology to bioengineer kidneys for transplantation, it also serves as a demonstration that cells have specific homing mechanisms which are directed by molecular determinants on the basement membranes of vessels and tubules. What is not clear from the above in vivo studies is whether there 
is merely engraftment of the instilled cells or whether, after engraftment, these cells multiply to cover the denuded areas.

\section{Conclusions}

The ideas expressed here are intended as a means of broadening the discussion on the important issue of how to halt disease progression in a solid organ and stimulate regeneration and/or restoration of function simultaneously. Most of the suggestions outlined require experimental confirmation in animal models well before they become applicable in humans with hypoxic kidneys [17]. When the possibility of applying the suggested vasculogenic strategy in humans becomes actual, it will be imperative to start with a much better insight into the in vivo pathology of the diseased kidney than we currently have. In particular, we will need to be able to visualize hypoxic regions, the vascular flow at the capillary level, energy metabolism, and other parameters using noninvasive techniques in human patients. Ultimately, if an intervention can be introduced in the appropriate patient at the appropriate point in the disease trajectory, renal regeneration may become a standard option for patients with CKD.

\section{References}

1 Oliver J: Architecture of the Kidney in Chronic Bright's Disease. New York, Hoeber, 1939.

- 2 Fine LG, Orphanides C, Norman J: Progressive renal disease: the chronic hypoxia hypothesis. Kidney Int 1998;53:S74-S78.

- 3 Fine LG, Norman JT: Chronic hypoxia as a mechanism of progression of chronic kidney diseases: from hypothesis to novel therapeutics. Kidney Int 2008;74:867-872.

4 Norman JT, Fine LG: Intrarenal oxygenation in chronic renal failure. Clin Exp Pharmacol Physiol 2006;33:989-996.

5 Norman J, Stidwell R, Singer M, Fine LG: Angiotensin II blockade augments renal cortical $\mathrm{pO}_{2}$ indicating a novel, potentially renoprotective action. Nephron Physiol 2003;94:3946.

6 Mimura I, Nangaku M: The suffocating kidney: tubulointerstitial hypoxia in end-stage renal disease. Nat Rev Nephrol 2010;11:667678.

7 Yasuda K, Pork HC, Ratliff B, Addabbo F, Hazopoulos AK, Chander P, Goligorsky MS: Adriamycin nephropathy, a failure of endothelial progenitor cell-induced repair. Am J Pathol 2010;176:1685-1695.
8 Fine LG: First heal thyself: rescue of dysfunctional endothelial progenitor cells restores function to the injured kidney. Am J Path 2010;176:1586-1587.

-9 Long DA, Norman JT, Fine LG: Restoring the renal microvasculature to heal the diseased kidney. Nat Rev Nephrol 2012;8:242-250.

10 Bussolati B, Bruno S, Grange C, Buttiglieri S, Deregibus MC, Cantino D, Camussi G: Isolation of renal progenitor cells from adult human kidney. Am J Pathol 2005; 166:545-555.

11 Chen J, Park HC, Addabbo F, Ni J, Pelger E, Li H, Plotkin M, Goligorsky MS: Kidney-derived mesenchymal stem cells contribute to vasculogenesis, angiogenesis and endothelial repair. Kidney Int 2008;74:879-889.

12 Sangidorj O, Yang SH, Jang HR, Lee JP, Cha RH, Kim SM, Lim CS, Kim YS: Bone marrowderived endothelial progenitor cells confer renal protection in a murine chronic renal failure model. Am J Physiol Renal Physiol 2010; 229:F325-F335.
13 Chade AR, Zhu X, Lavi R, Krier JD, Pislaru S, Simari RD, Napoli C, Lerman A, Lerman OL: Endothelial progenitor cells restore renal function in chronic experimental renovascular disease. Circulation 2009;119:547-557.

14 Goligorsky MS, Yasuda K, Ratliff B: Dysfunctional endothelial progenitor cells in chronic kidney disease. J Am Soc Nephrol 2010;21: 911-919.

15 Yoon CH, Koyanagi M, Iekushi K, Seeger F, Urbich C, Zeiher AM, Dimmeler S: Mechanism of improved cardiac function after bone marrow mononuclear cell therapy: role of cardiovascular lineage commitment. Circulation 2010;121:2001-2011.

-16 Song JJ, Guyette JP, Gilpin SE, Gonzalez G, Gabriel G, Vacanti JP, Ott HC: Regeneration and experimental orthotopic transplantation of a bioengineered kidney. Nat Med 2013;19: 646-651.

17 Fine LG, Dharmakumar R: Limitations of BOLD-MRI for assessment of hypoxia in chronically diseased human kidneys. Kidney Int 2012;82:934-935.
Restoring the Function of a Diseased Kidney via Its Microvasculature 